\title{
Intercomparison of three indices for addressing drought variability in North China Plain during 1962-2012
}

\author{
HONGJIAN LU ${ }^{1,2}$, XINGGUO MO ${ }^{1}$ \& SUXIA LIU ${ }^{1}$ \\ 1 Key Laboratory of Water Cycle and Related Land Surface Processes, Institute of Geographic Sciences and Natural \\ Resources Research, Chinese Academy of Sciences, Beijing 100101, China \\ 2 University of Chinese Academy of Sciences, Beijing100049, China \\ moxg@igsnrr.ac.cn
}

\section{INTRODUCTION}

Drought is one of the most complex climatic phenomena causing detrimental damage to the society and environment. Although a universal definition of drought is still lacking, it is usually related to a long and sustained period during which water becomes scarce. Compared with other hydrological hazards, such as floods, the development of droughts is slow and the inception and termination of droughts are very difficult to identify. Moreover, droughts are only perceived when human activities and the environment are affected. Thus, droughts are the best example of a "creeping and penetrating" type natural hazard (Gillette 1950, Wilhite et al. 1985, 2005).

The North China Plain (NCP), one of the huge granaries and the centre of politics, economics and culture in China, has frequently been subjected to droughts during recent decades. With population growth and agriculture expansion, the sustainable development of NCP has been challenged by constrained water resources and associated disasters. In other words, NCP is turning into one of the most vulnerable "hotspots" in China in terms of water shortage and water security. Accurate assessments of drought for an early warning would play a critical role in water resources management and planning. The present study compares the spatio-temporal variations of droughts in NCP by using three commonly-used indices and aims to elucidate the spatiotemporal patterns of drought and explore a relative, reasonable index for describing regional water security in NCP.

\section{METHOD}

The three indices we used are the Standardized Precipitation Index (SPI, McKee et al. 1993), which is solely based on a precipitation record, the Standardized Precipitation-Evapotranspiration Index (SPEI, Vicente-Serrano et al. 2010), which integrates temperature into SPI for the calculation of potential evapotranspiration, and the Palmer Drought Severity Index (PDSI, Palmer 1965), which derives the total moisture status of a region by a two-layer soil water balance model based on precipitation and temperature for estimating moisture supply and demand. The PDSI used here is the self-calibrating PDSI (SC-PDSI) by Wells et al. (2004), which adopts the dynamically calculated weighting factors but not the empirical constants, enabling more credible comparisons of drought severity for different locations.

Daily precipitation and temperature at 66 meteorological stations in NCP obtained from the China Meteorological Administration were used for calculating the three indices. Homogeneity tests were conducted with daily precipitation at each station. Then the daily precipitation and temperature were aggregated into monthly sum and monthly mean values, respectively, to serve as input to the calculations of the drought indices for the period 1962-2012.

\section{RESULTS}

\section{Temporal evolution of drought and wetness}

As shown in Fig. 1(I), overall, drought severity has significantly increased with small periodical shifts between wet and dry spells. As drought is both related with water supply (precipitation) and energy (temperature), it is seen that the PDSI and SPEI better described the wet period around 1964 and drought extreme around 1999, respectively, than the SPI does.

The M-K test shows the year 1975/1976 is the primary abrupt change point (Fig. 1(II)) with all the indices. However, PDSI and SPEI show an obvious decreasing trend, while for SPI this is 
not so obvious, once again showing the exact overlap role of precipitation and temperature on PDSI or SPEI. SPI did show a secondary turning point around 2006, indicating wetter years then, but the signal not so strong. The signal of the point at around 2010 changing to wet is even weaker. The popular view that there was a so-called big shift from north drying to north wetness starting in 2010 (Gong et al. 2013) cannot be supported by our result.

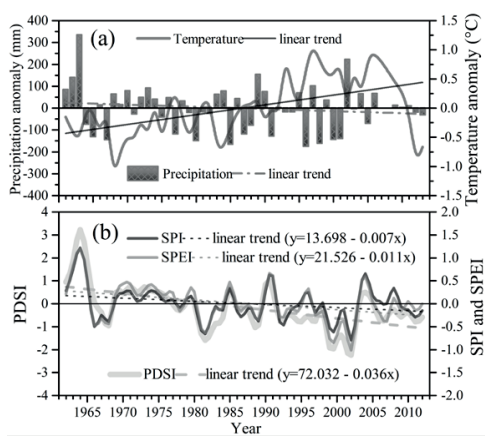

Fig. 1 (I) Evolution of drought and wetness phases

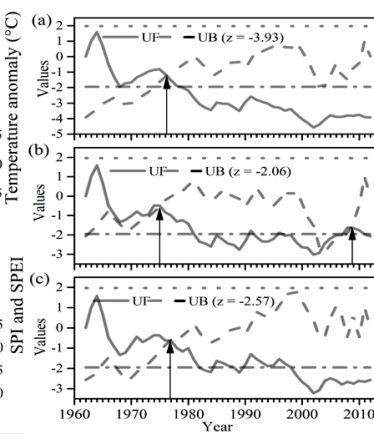

(II) Results of M-K methods for

(a) PDSI, SPI (b) and SPEI (c)

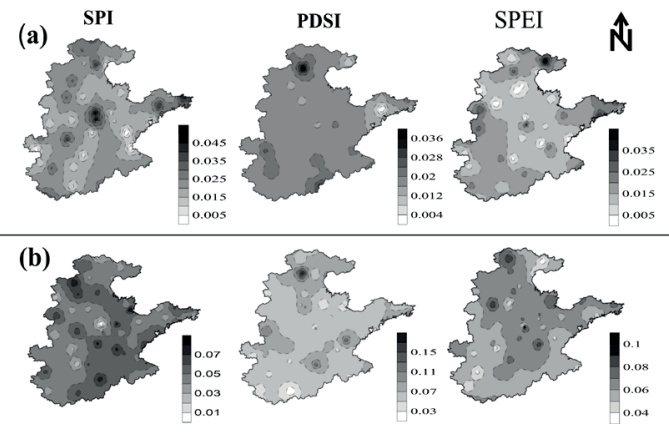

(III) Spatial patterns of extreme (a) and severe (b) drought frequencies

\section{Spatial patterns of drought variability}

There are some differences between the spatial distributions of drought frequencies derived from the three indices (Fig. 1(III)). The spatial pattern of drought variability is fragmented and ragged rather than a simple "north-south" dipole as imagined by common sense. For SPI and SPEI, extreme droughts (value $\leq-2.0$ ) mainly happened frequently in the northwest and east of Shandong province, while that of PDSI $(\leq-4.0)$ in the north of Hebei province. Concurrently, distributions of severe drought $(-4.0<$ PDSI $<-3.0,-2.0<$ SPEI and SPI $<-1.5)$ are different among the three indices, and all have fragmented patterns. In other words, local differences of drought occurrences among the three indices are ubiquitous.

\section{CONCLUSIONS}

SPI, SPEI and PDSI have good performance in detecting drought in NCP, but the latter two are relatively more direct role in displaying wet-dry patterns than the SPI. Transitions between dry and wet spells are essentially caused by precipitation anomalies, whereas temperature increase can accelerate the drying process, resulting in intensified magnitudes of drought characteristics.

The spatiotemporal characteristics of drought in NCP are complex and changeable. A more comprehensive index with the consideration of human activity (e.g. irrigation ratio) is expected to display drought better for water resources management and planning.

Acknowledgements This work was jointly supported by the key project for the strategic science plan in IGSNRR, CAS (2012ZD003), the National Natural Science of China (No. 31171451 and 41471026) and the National Basic Research Programme of China (No. 2010CB428404).

\section{REFERENCES}

Gillette, H. (1950) A creeping drought under way. Water and Sewage Works (March), 104-105.

Gong, Z., et al. (2013) Analysis of the summer precipitation of 2012 in East China and its possibility of decal shift. Acta Phys. Sin. 62, No. 9009205 (in Chinese).

McKee, T. et al. (1993) The relationship of drought frequency and duration to time scale. AMS, Anaheim, Calif. Palmer, W. (1965) Meteorological drought. US Department of Commerce Weather Bureau, Washington, DC.

Vicente-Serrano, S., et al. (2010) A multiscalar drought index sensitive to global warming: the standardized precipitation evapotranspiration index. Journal of Climate (23), 1696-1718.

Wells, N., et al. (2004) A self-calibrating Palmer Drought Severity Index. Journal of Climate (17), 2335-2351.

Wilhite, D. (ed.) 2005) Drought and Water Crises: Science, Technology and Management Issues. CRC Press, 406 pp.

Wilhite, D., et al. (1985) Understanding the drought phenomenon: the role of definitions. Water International 10, 111-120. 